\title{
MAXIMAL SINGULAR INTEGRAL TRANSFORMS ON LOCAL FIELDS
}

\author{
JIA-ARNG CHAO
}

\begin{abstract}
We show that, on local fields, "nice" singular integral transforms preserve $H^{p}$-spaces for $0<p<\infty$ where $H^{p}$ is the space of all distributions whose maximal functions are in $L^{p}$. A version of the $\mathrm{F}$. and M. Riesz theorem is also obtained.
\end{abstract}

1. By a local field we mean a locally compact, nondiscrete, totally disconnected (complete) field. Such a field is a $p$-adic field, a finite algebraic extension of a $p$-adic field, or a field of formal Laurent series over a finite field. The Calderón-Zygmund type of singular integral transforms on local fields were treated in [4] and [8]. The theory of $H^{p}$-spaces of conjugate systems in the sense of Stein-Weiss was studied in [2] and [1]. A more complete list of references of various results on harmonic analys is on local fields can be found in [1]. We shall assume some familiarities of notation and preliminaries in [1].

Let $K$ be a fixed local field with the ring of integers $\mathcal{C} . \mathcal{C} / \mathcal{P} \cong G F(q)$ where $\mathcal{P}$ is the (unique) maximal ideal in $\mathcal{C}$ and $q$ is a prime power. Let $p$ be a generator of $\mathcal{P},|\boldsymbol{p}|=q^{-1}$. For $k \in \mathbf{Z}$, let $\mathcal{P}-k=\left\{x \in K:|x| \leq q^{k}\right\}$, $\left(\mathcal{O}=\mathcal{P}^{0}\right) \cdot \mathcal{P}_{y}^{-k}=y+\mathcal{P}^{-k}$ are spheres. The Haar measure on $K$ has been normalized so that $|\mathfrak{O}|=\int_{\mathcal{O}} d x=1$ and $\left|\mathcal{P}_{y}^{-k}\right|=q^{k}$ for all $k$.

Let $\pi$ be a (inultiplicative) unitary character on $K^{*}$ such that it is homogenous of degree 0 and is ramified of degree $b \geq 1$ (i.e., $\pi\left({ }_{p}^{k} x\right)=\pi(x)$ for all $k \in \mathbf{Z}$ and $\pi$ is constant on $\mathcal{P}_{1}^{h}$ but not on $\left.\overline{\mathcal{P}}_{1}^{-1}\right)$. $\pi$ is, thus, constant on cosets of $\mathcal{P}-m+h$ in $\mathcal{P}-m \backslash \mathcal{P}-m+1=\left\{x \in K:|x|=q^{m}\right\}$ and $\left.\int_{\{|x|=q} m\right\}^{\pi}(x) d x=0$ for all $m \in \mathbf{Z}$. Denote $Q(x)=c_{\pi}\left(\pi(x) /|x|\right.$ ) where $c_{\pi}=1 / \Gamma(\pi)$. (See [5] for details.) Distributions on $K$ have been identified with regular functions on $K \times \mathbf{Z}$. The regularization kernel $R_{k}(x)=q^{-k} \Phi_{-k}(x)$, where $\Phi_{-k}$ is the

Received by the editors February 27, 1974.

AMS (MOS) subject classifications (1970). Primary 44A25, 12B99, 46J15; Secondary $43 \mathrm{~A} 70$.

Key words and phrases. Local field, singular integral transform, maximal operator, $H^{p}$-space. 
characteristic function of $\mathcal{P}^{-k}$, serves as the Poisson kernel. (See [7] and [1] for details.) It is easy to see that $R_{n} * Q=Q\left[1-\Phi_{-(n+h-1)}\right]$. Let $Q_{n}=R_{n} * Q$ and $Q_{n}^{N}=Q_{n} \Phi_{-N}$ for $N \geq n+h$. We note that $Q_{n}^{N} * f(x, k)=$ $Q_{k}^{N} * f(x, k)=Q^{N} * f(x, k)$ for $n \leq k \leq N-b$ if $f(x, k)$ is regular on $K \times \mathbf{Z}$.

For a distribution $f$ on $K$ (i.e., $f(x, k)=\left(R_{k} * f\right)(x)$, a regular function on $K \times \mathbf{Z}$ ), define

$$
\left(T_{\pi} f\right)(x, k)=\lim _{N \rightarrow \infty} Q^{N} * f(x, k) \quad \text { for }(x, k) \in K \times \mathbf{Z} .
$$

For "nice" functions $f$ on $K$, this is just a sort of singular integral transform as was studied in [4], [8] and [1]. In particular, if $f \in L^{p}(K)$ with $1 \leq p<\infty$, then $\left(T_{\pi} f\right)(x, k)=T_{\pi}(f(x, k))$ is a well-defined regular function on $K \times \mathbf{Z}$ and $T_{\pi} f(x)=\lim _{k \rightarrow-\infty}\left(T_{\pi} f\right)(x, k)$ exists almost everywhere. Moreover $T_{\pi}$ is of weak-type $(1,1)$ and of type $(p, p)$ for $1<p<\infty$. It is obvious that the maximal operator

$$
f \rightarrow f^{*}(x)=\sup _{k \in Z}|f(x, k)|=\sup _{k \in Z}\left|R_{k} * f(x)\right|
$$

as well as the Hardy-Littlewood maximal operator $f \rightarrow M f(x)=\sup _{k \in Z} R_{k} *|f|(x)$ is also of weak-type $(1,1)$ and of type $(p, p)$ for $1<p<\infty$.

For $0<p<\infty$, let $H^{p}(K)$ be the space of all distributions $f$ on $K$ whose maximal function $f^{*} \in L^{p}(K)$ with the "norm" $\|f\|_{H^{p}}=\left\|f^{*}\right\|_{p}$. This way of defining $H^{p}$ is suggested by the results of Burkholder-Gundy-Silverstein and Fefferman-Stein (see e.g. [3]). Hence, $H^{p}=L^{p}$ if $1<p<\infty$ and $H^{1} \subset L^{1}$. Indeed, if $1<p<\infty$, since $f=T_{\pi^{-1}}\left(T_{\pi} f\right)$, we have

$$
A_{p}\|f\|_{p} \leq\left\|T_{\pi} f\right\|_{p} \leq B_{p}\|f\|_{p} \text { with constants } A_{p}, B_{p}>0
$$

and

$$
\|f\|_{p} \leq\left\|f^{*}\right\|_{p} \leq p^{\prime}\|f\|_{p} \quad \text { where } 1 / p+1 / p^{\prime}=1 \text {. }
$$

In other words, $\left\|f^{*}\right\|_{p} \approx\|f\|_{p} \approx\left\|T_{\pi} f\right\|_{p} \approx\left\|\left(T_{\pi} f\right)^{*}\right\|_{p}$ if $1<p<\infty$. Our main result is to show that $T_{\pi}$ preserves $H^{p}$ for $0<p<\infty$. That is, for $0<p<\infty$,

$$
\left\|\left(T_{\pi} f\right)^{*}\right\|_{p} \leq C_{p}\left\|f^{*}\right\|_{p} \text { for some } C_{p}>0
$$

and hence

$$
\left\|f^{*}\right\|_{p} \approx\left\|\left(T_{\pi} f\right)^{*}\right\|_{p} \text { for } 0<p<\infty
$$

A sharper version of the F. and M. Riesz theorem on local fields (than that of [1]) is obtained as a consequence. 
2. We have only to consider the case $0<p \leq 1$. Let $f \in H^{p}$, i.e., $f(x, k)$ is a regular function on $K \times \mathbf{Z}$ such that $f^{*}(x)=\sup _{k}|f(x, k)| \in L^{p}$. We first show that $\left(T_{\pi} f\right)(x, k)$ exists for all $x$ and all $k$.

For $\lambda>0$, the set $\left\{x \in K: f^{*}(x)>\lambda\right\}$ can be written as the disjoint union of spheres $\left\{P_{x_{j}}^{-\left(l_{j}-1\right)}\right\}_{j=1}^{\infty}$ such that $\left|f\left(x_{j}, l_{j}-1\right)\right|>\lambda \geq\left|f\left(x_{j}, l_{j}\right)\right|$. For a sphere $S=\mathscr{P}_{x}^{-l}$, let $S^{h}=\mathscr{P}_{x}^{-(l+h-1)}$. Let $D_{\lambda}=\bigcup_{j=1}^{\infty} \mathcal{P}_{x_{j}}^{-l_{j}}=\bigcup_{t=1}^{\infty} S_{t}$ where the spheres $S_{t}=P_{x}^{-l_{j}}, t=1,2, \cdots$, are mutually disjoint, and $D_{\lambda}^{h}=$ $\bigcup_{t=1}^{\infty} s_{t}^{h}$. Hence,

$$
\left|D_{\lambda}\right| \leq \sum_{t=1}^{\infty}\left|S_{t}\right|=q\left|\left\{f^{*}>\lambda\right\}\right| \text { and } \quad\left|D_{\lambda}^{h}\right| \leq q^{h-1}\left|D_{\lambda}\right| \text {. }
$$

For a fixed $k \in \mathbf{Z}$, let $D_{\lambda k}=\bigcup_{t} S_{t k}$ where $\left\{S_{t k}\right\}$ are those spheres $S_{t}=P_{x_{j}}^{-l_{j}}$ such that $l_{j}>k$, and $D_{\lambda k}^{h}=\bigcup_{t} S_{t k}^{h}$.

Let $g(x, k)=f\left(x_{j}, l_{j}\right)$ if $\mathscr{P}_{x}^{-k} \subset S_{t}=\mathscr{P}_{x_{j}}^{-l_{j}}$ and $g(x, k)=f(x, k)$ otherwise. Then $g(x, k)$ is regular on $K \times \mathbf{Z},|g(x, k)|^{j} \leq \lambda$ and $|g(x, k)| \leq f^{*}(x) \in L^{p}$. Thus $g(x, k) \in L^{2}$ for all $k$. Moreover, $g(x)=\lim _{k \rightarrow-\infty} g(x, k)$ exists a e. and is in $L^{2}$. Write $b(x, k)=f(x, k)-g(x, k)$ for all $x$ and all $k$. We have that $\int_{S_{t k}} b(x, k) d x=0$ for all $S_{t k}$ in $D_{\lambda k}$, also that $b(x, k)=0$ if $\mathcal{P}_{x}^{-k} \not \subset$ $D_{\lambda k}$, i.e., $x \notin D_{\lambda k}$.

Now, for fixed $k \in \mathbf{Z}$ and for large $N$ such that $N \geq k+h$ and $\left|D_{1}\right|<q^{N}$,

$$
\begin{aligned}
Q^{N} * b(x, k) & =\int_{\mathcal{\rho}}^{\prime} Q_{k}(y) b(x-y, k) d y \\
& =\int_{\mathcal{P}_{x}^{-N}} Q_{\mathrm{ND}_{\lambda k}}(x-y) b(y, k) d y=\sum \int_{S_{t k}} Q_{k}(x-y) b(y, k) d y
\end{aligned}
$$

where the summation is taken over all spheres $S_{t k}$ in $D_{\lambda k}$ such that $S_{t k} C$ $\mathcal{P}_{x}^{-N}$. (Note that the sphere $\mathcal{P}_{x}^{-N}$ is larger than any sphere $S_{t k}$ in $D_{\lambda k}$, so if $S_{t k}^{x} \cap \mathscr{P}_{x}^{-N} \neq \varnothing$, then $\left.S_{t k} \subset \mathscr{P}_{x}^{x}-N_{0}\right)$ If $x \notin D_{\lambda k}^{h}$ and $y \in S_{t k}=\mathscr{P}_{x_{t}}{ }^{-} l_{t}$, then $\left|x-x_{t}\right|^{x}=q^{m} \geq q^{l_{t}+h}$ and $\left|(x-y)-\left(x-x_{t}\right)\right|^{t k}=\left|y-x_{t}\right| \leq q^{l} t \leq q^{m-h}$. Since $Q_{k}$ is constant on cosets of $\mathcal{P}-m+b$ in $\left\{x:|x|=q^{m}\right\}$, we see that $Q_{k}(x-y)$ $=Q_{k}\left(x-x_{t}\right)$. From the fact that $\int_{S_{t k}} b(y, k) d y=0$, it follows that $Q^{N} *$ $b(x, k)=0$ for large $N$ and if $x \notin D_{\lambda k}^{h} \subset D_{\lambda}^{h}$.

Since $g(x, k) \in L^{2}$ for all $k, Q^{N} * g(x, k)$ is well defined for all $x$ so is $\left(T_{\pi} g\right)(x, k)$. Moreover, $\left(T_{\pi} g\right)(x, k) \in L^{2}$ for all $k$. Hence, for any fixed $k$, $Q^{N} * f(x, k)=Q^{N} * g(x, k)+Q^{N} * b(x, k)$ is defined for all large $N$ and for all $x$, except possibly for $x \in D_{\lambda k}^{h}$. Since $f^{*} \in L^{p}$, we have $\left|D_{\lambda}\right| \rightarrow 0$ as $\lambda \rightarrow \infty$. Hence $\left|D_{\lambda k}^{h}\right|=0$ for sufficiently large $\lambda$. Therefore $\left(T_{\pi} f\right)(x, k)=$ $\lim _{N \rightarrow \infty} Q^{N} * f(x, k)$ is defined for all $x$. 
It is easy to see that $\left(T_{\pi} f\right)(x, k)$ is a regular function on $K \times \mathbf{Z}$. Call the corresponding distribution on $K, T_{\pi} f$. We note that, by comparing the Fourier transforms, the regularization of the distribution $T_{\pi} f$ is just $\left(T_{\pi} f\right)(x, k)$. It remains to show that $\left(T_{\pi} f\right)^{*} \in L^{p}$.

Lemma. For $\lambda>0$, let $E_{\lambda}=\left\{x \in K:\left(T_{\pi} f\right)^{*}(x)>2 \lambda\right\}$.

$$
\left|E_{\boldsymbol{\lambda}}\right| \leq A \lambda^{-2} \int_{0}^{\lambda}\left|D_{s}\right| s d s \text { for some constant } A>0 \text {. }
$$

Proof. Write $f(x, k)=g(x, k)+b(x, k)$ as above. Since

$$
\begin{aligned}
\left(T_{\pi} f\right)^{*}(x) & =\sup _{k}\left|\left(T_{\pi} f\right)(x, k)\right| \\
& \leq \sup _{k}\left|\left(T_{\pi} g\right)(x, k)\right|+\sup _{k}\left|\left(T_{\pi} b\right)(x, k)\right|=\left(T_{\pi} g\right)^{*}(x)+\left(T_{\pi} b\right)^{*}(x),
\end{aligned}
$$

we have

$$
\left|E_{\lambda}\right|=\left|\left\{\left(T_{\pi} f\right)^{*}>2 \lambda\right\}\right| \leq\left|\left\{\left(T_{\pi} g\right)^{*}>\lambda\right\}\right|+\left|\left\{\left(T_{\pi} b\right)^{*}>\lambda\right\}\right| \text {. }
$$

It follows from the $L^{2}$-theory, since $g(x)=\lim _{k \rightarrow \infty} g(x, k) \in L^{2}$, that $\left|\left\{\left(T_{\pi} g\right)^{*}>\lambda\right\}\right| \leq 2 \lambda^{-2}\left\|T_{\pi} g\right\|_{2}^{2} \leq 2 B \lambda^{-2}\|g\|_{2}^{2} \leq 2 B \lambda^{-2} \int_{x \notin D_{\lambda}}\left(f^{*}(x)\right)^{2} d x+2 B\left|D_{\lambda}\right| \cdot$ Moreover,

$$
\begin{aligned}
\lambda^{-2} \int_{x \in D_{\lambda}}\left(f^{*}(x)\right)^{2} d x & \leq \lambda^{-2} \int_{\left\{f^{*} \leq \boldsymbol{\lambda}\right\}}\left(f^{*}(x)\right)^{2} d x \\
& =\lambda^{-2} \int_{0}^{\lambda}\left|\left\{f^{*}>s\right\}\right| 2 s d s \leq 2 \lambda^{-2} \int_{0}^{\lambda}\left|D_{s}\right| s d s,
\end{aligned}
$$

and

$$
\left|D_{\lambda}\right|=\left|D_{\lambda}\right| 2 \lambda^{-2} \int_{0}^{\lambda} s d s \leq 2 \lambda^{-2} \int_{0}^{\lambda}\left|D_{s}\right| s d s
$$

Hence, we have

$$
\left|\left\{\left(T_{\pi} g\right)^{*}>\lambda\right\}\right| \leq 4 B \lambda^{-2} \int_{0}^{\lambda}\left|D_{s}\right| s d s
$$

We have shown that $\left(T_{\pi} b\right)(x, k)=0$ if $x \notin D_{\lambda k}^{h} \subset D_{\lambda}^{b}$. Thus, $\left(T_{\pi} b\right)^{*}(x)=$ $\sup _{k}\left|\left(T_{\pi} b\right)(x, k)\right|=0$ if $x \notin D^{h}$. Therefore

$$
\left|\left\{\left(T_{\pi} b\right)^{*}>\lambda\right\}\right| \leq\left|D_{\lambda}^{h}\right| \leq q^{h-1}\left|D_{\lambda}\right| \leq q^{h-1} 2 \lambda^{-2} \int_{0}^{\lambda}\left|D_{s}\right| s d s .
$$

(3), (4) and (5) give us the estimate (2). The Lemma is proved.

Now, from (2) and (1), we have $\left|E_{\lambda}\right| \leq A q \lambda^{-2} \int_{0}^{\lambda}\left|\left\{f^{*}>s\right\}\right| s d s$. Thus, by a usual integration argument with $0<p \leq 1$, 


$$
\begin{aligned}
\int_{0}^{\infty} \lambda^{p-1}\left|E_{\lambda}\right| d \lambda & \leq A q \int_{0}^{\infty} \lambda^{p-3} \int_{0}^{\lambda}\left|\left\{f^{*}>s\right\}\right| s d s d \lambda \\
& =A q \int_{0}^{\infty} \int_{s}^{\infty} \lambda^{p-3} d \lambda\left|\left\{f^{*}>s\right\}\right| s d s \\
& =\frac{A q}{2-p} \int_{0}^{\infty} s^{p-1}\left|\left\{f^{*}>s\right\}\right| d s=\frac{A q}{p(2-p)}\left\|f^{*}\right\|_{p}^{p} .
\end{aligned}
$$

Hence, $\left\|\left(T_{\pi} f\right)^{*}\right\|_{p} \leq C_{p}\left\|f^{*}\right\|_{p}$ where $C_{p}$ depends only on $\pi, q$ and $p$.

Therefore we have shown the following

Theorem. For $0<p<\infty, f \in H^{p}$ implies $T_{\pi} f \in H^{p}$ and, moreover, $\|f\|_{H^{p}}$ $\approx\left\|T_{\pi} f\right\|_{H}$.

Remark. With some slight modifications the above argument also applies to "nice" singular integral transforms on $K^{n}$, the $n$-dimensional vector space over $K$, and to multiplier transforms $T$ on $K^{n}$ such that $(T f)^{\wedge}=m \hat{f}$ with $m$ satisfying some smooth conditions, in particular, those cases studied in $[8, \S 4]$.

3. Let us consider the most interesting case, $p=1$. The following result is a (sharper) version of the F. and M. Riesz theorem:

Theorem. Suppose $\mu_{0}, \mu_{1}, \cdots, \mu_{m}$ are finite Borel measures on $K$. If $F(x, k)=\left(\mu_{0}(x, k), \mu_{1}(x, k), \cdots, \mu_{m}(x, k)\right)$ forms a "conjugate system", then each $\mu_{j}$ is absolutely continuous and $\mu_{j}^{*} \in L^{1}, j=0,1, \cdots, m$. Moreover, $T_{\pi} \mu_{j}, j=0,1, \ldots, m$, is also absolutely continuous for any "nice" singular integral transform $T_{\pi}$ (as described earlier).

Proof. The first part of the theorem was proved in [1]. From our main result, since $\mu_{j}^{*} \in L^{1}$, we have $\left(T_{\pi} \mu_{j}\right)^{*} \in L^{1}$. Hence, $\left(T_{\pi} \mu_{j}\right)(x, k)$ is the regularization of an $L^{1}$-function. Therefore, $T_{\pi} \mu_{j}$ is absolutely continuous.

The set $\bigcup_{k=-\infty}^{\infty} p^{k}(\epsilon+\mathcal{P})$, where $\epsilon+\mathscr{P}$ is a coset of $\mathcal{P}$ in $\{|x|=1\}$, is said to be a "cone". As a corollary, we have another version of the Riesz brothers' theorem:

Theorem. Suppose $\mu$ is a finite Borel measure on $K$ such that $\hat{\mu}$, the Fourier transform of $\mu$, is supported on a "cone". Then $\mu$ is absolutely continuous, so is $T_{\pi} \mu$.

As a final remark we consider the case $p=1$ for the Hardy-Littlewood. maximal operator $f \rightarrow M f=\sup _{k} R_{k} *|f|$. We show the following local field variant of a result of Stein [6]: 
Theorem. Suppose $f$ is an $L^{1}$-function which is supported on some sphere S. Then $\int_{S} M f(x) d x<\infty$ if and only if $\int_{S}|f(x)| \log ^{+}|f(x)| d x<\infty$.

Proof. The fact that $\int_{S}|f| \log { }^{+}|f| d x<\infty$ implies $\int_{S} M f d x<\infty$ is known (e.g., [4]) just as its well-known analogue in the $\mathbf{R}^{n}$ setting. With some modifications, the converse can be similarly shown.

For $\lambda>0$, let $F_{\lambda}=\{x: M f(x)>\lambda\}$. Write $F_{\lambda}=\bigcup_{t=1}^{\infty} S_{t}$ where $\left\{S_{t}\right\}_{t=1}^{\infty}$ are mutually disjoint spheres such that

$$
\frac{1}{\left|S_{t}\right|} \int_{S_{t}}|f(x)| d x>\lambda \geq \frac{1}{\left|S_{t}^{2}\right|} \int_{S_{t}^{2}}|f(x)| d x .
$$

Let $A=\int_{S}|f| d x$. For $x \notin S$, since $f$ is supported on $S$, we have $M f(x)$ $<A$. Thus, if $\int_{S} M f d x<\infty$, then $\int_{B} M f d x<\infty$ for any finite sphere $B$. Hence, $\int_{F_{\alpha}} M f d x<\infty$ for all $\alpha>0$. Consequently, $\int_{\alpha}^{\infty}\left|F_{\lambda}\right| d \lambda<\infty$ for any $\alpha>0$. Now,

$$
\left|F_{\lambda}\right|=\sum_{t}\left|S_{t}\right|=q^{-1} \sum_{t}\left|S_{t}^{2}\right| \geq q^{-1} \lambda^{-1} \sum_{t} \int_{S_{t}^{2}}|f| d x \geq q^{-1} \lambda^{-1} \int_{F_{\lambda}}|f| d x .
$$

Therefore, by a usual integration argument, it follows that $\int_{S}|f| \log ^{+}|f| d x$ $<\infty$.

We note that $M f \in L^{1}(K)$ implies $f \in L \log ^{+} L(K)$, while the converse implication fails.

The author profited from several conversations with R. R. Coifman and M. H. Taibles on on the contents of this note.

\section{REFERENCES}

1. J.-A. Chao, $H^{p}$-spaces of conjugate systems on local fields, Studia Math. 49 (1974), 267-287.

2. J.-A. Chao and M. H. Taibleson, A subregularity inequality for conjugate systems on local fields, Studia Math. 46 (1973), 249-257.

3. C. Fefferman and E. M. Stein, $H^{p}$-spaces of several variables, Acta Math. 129 (1972), 137-193.

4. K. Phillips and M. H. Taibleson, Singular integrals in several variables over a local field, Pacific J. Math. 30 (1969), 209-231. MR 40 \#7886

5. P. J. Sally, Jr. and M. H. Taibleson, Special functions on locally compact fields, Acta Math. 116 (1966), 279-309. MR 34 \#6168.

6. E. M. Stein, Note on the class L $\log L$, Studia Math. 32 (1969), 305-310. MR 40 \#799

7. M. H. Taibleson, Harmonic analysis on $n$-dimensional vector spaces over local fields, II. Generalized Gauss kernels and the Littlewood-Paley function, Math. Ann. 186 (1970), 1-19. MR 41 \#8989

8. - Harmonic analysis on n-dimensional vector spaces over local fields, III. Multipliers, Math. Ann. 187 (1970), 259-271. MR $42 \# 8178$. 